\title{
A coupling method involving the Sumudu transform and the variational iteration method for a class of local fractional diffusion equations
}

\author{
Feng Gao $o^{a, b, *}$, H. M. Srivastava ${ }^{c, d}$, Ya-Nan Gao ${ }^{a, b}$, Xiao-Jun Yang ${ }^{a, b}$ \\ ${ }^{a}$ School of Mechanics and Civil Engineering, China University of Mining and Technology, Xuzhou 221116, P. R. China. \\ ${ }^{b}$ State Key Laboratory for Geomechanics and Deep Underground Engineering, School of Mechanics and Civil Engineering, China \\ University of Mining and Technology, Xuzhou 221116, P. R. China. \\ ${ }^{c}$ Department of Mathematics and Statistics, University of Victoria, Victoria, British Columbia V8W 3R4, Canada. \\ ${ }^{d}$ Department of Medical Research, China Medical University Hospital, China Medical University, Taichung 40402, Taiwan, P. R. \\ China.
}

Communicated by D. Baleanu

\begin{abstract}
In this article, a coupling of the variational iteration method with the Sumudu transform via the local fractional calculus operator is proposed for the first time. As a testing example, the exact solution for the local fractional diffusion equation in fractal one-dimensional space is obtained. The method provided an accurate and efficient technique for solving the local fractional PDEs. (C)2016 All rights reserved.
\end{abstract}

Keywords: Diffusion equation, exact solution, variational iteration method, Sumudu transform, local fractional calculus.

2010 MSC: 35R11, 26A33, 35A15.

\section{Introduction}

Fractional heat transfer (see [1 15, 8, 9, 11, 13, 15, 18, 19, 22]) is an abnormal phenomenon of dynamical systems to capture the relations in space and time with different kernels of non-differentiable and differentiable types. This interest spans the works of many scientists and engineers from the field of mathematical

\footnotetext{
${ }^{*}$ Corresponding author

Email address: jsppw@sohu.com (Feng Gao)
} 
physics. In order to find the solutions of the fractional heat transfer problems, several methods, such as the heat-balance integral method (HBIM) [6], the Green function method (GFM) [7, the similarity variable method (SVM) [21, the variational iteration method (VIM) [20] and the Laplace transform variational iteration method (LTVIM) [10], were developed in recent years.

In this paper, we consider the local fractional diffusion equation in fractal one-dimensional space for description of the fractal heat transfer (see [10, 20, 22]):

$$
\frac{\partial^{\kappa} \Theta(x, t)}{\partial \tau^{\kappa}}-\frac{\partial^{2 \kappa} \Theta(x, t)}{\partial x^{2 \kappa}}=0, \quad \kappa \in(0,1),
$$

where the local fractional derivative (LFD) of $\Theta(\tau)$ of order $\kappa$ is given as follows (see, for example, [10, 16, 17, 20 23]):

$$
\Theta_{\tau}^{(\kappa)}\left(\tau_{0}\right)=\left.\frac{d^{\kappa} \Theta(\tau)}{d \tau^{\kappa}}\right|_{\tau=\tau_{0}}=\lim _{\tau \rightarrow \tau_{0}} \frac{\Delta^{\kappa}\left(\Theta(\tau)-\Theta\left(\tau_{0}\right)\right)}{\left(\tau-\tau_{0}\right)^{\kappa}},
$$

with the difference term given by [22]

$$
\Delta^{\kappa}\left(\Theta(\tau)-\Theta\left(\tau_{0}\right)\right) \cong \Gamma(1+\kappa) \Delta\left(\Theta(\tau)-\Theta\left(\tau_{0}\right)\right)
$$

The local fractional variational iteration method (LFVIM) was used to solve the local fractional PDEs in fractal-dimension space (see [20, 22]). Meanwhile, the local fractional Sumudu transform (LFST) was expressed by (see [17]):

$$
S_{\kappa}\{\varphi(\tau)\}=\frac{1}{\Gamma(1+\kappa)} \int_{0}^{\infty} E_{\kappa}\left(-h^{-\kappa} \tau^{\kappa}\right) \frac{\varphi(\tau)}{h^{\kappa}}(d \tau)^{\kappa}, \quad 0<\beta \leq 1,
$$

and its inverse transform was defined by (see [17]):

$$
S_{\kappa}^{-1}\left\{S_{\kappa}(\Theta(\tau))\right\}=\Theta(\tau), \quad 0<\kappa \leq 1,
$$

where the local fractional integral of $\varphi(\tau)$ of order $\kappa$ is defined by (see [17])

$$
{ }_{\tau_{0}} I_{\tau}^{(\kappa)} \varphi(\tau)=\frac{1}{\Gamma(1+\kappa)} \int_{\tau_{0}}^{\tau} \varphi(\tau)(d \tau)^{\kappa}=\frac{1}{\Gamma(1+\kappa)} \lim _{\Delta \tau \rightarrow 0} \sum_{j=0}^{j=N-1} \varphi\left(\tau_{j}\right)(\Delta \tau)^{\kappa}
$$

with the partitions of the interval $\left[\tau, \tau_{0}\right]$ are given by $\left(\tau_{j}, \tau_{j+1}\right)\left(j=0, \cdots, N-1, \Delta \tau=\tau_{j+1}-\tau_{j}\right)$.

Motivated essentially by the above results, our aim in the present article is to propose a coupling method of LFVIM and LFST for solving the local fractional diffusion equation in fractal one-dimensional space at the first time (see also [12]).

This article is structured as follows: In Section 2, a coupling method of LFVIM and LFST is analyzed. The non-differentiable solution of local fractional diffusion equation is given in Section 3 . Finally, Section 4 outlines our conclusions.

\section{Analysis of the method}

In this section, a coupling method of VIM with ST via the local fractional calculus operator is introduced.

In the local fractional differential operator, 1.1) can be written as follows:

$$
\mathrm{E}_{\kappa} \Theta-\Pi_{\kappa} \Theta=0
$$

where

$$
\mathrm{E}_{\kappa}=\partial^{\kappa} / \partial \tau^{\kappa},
$$


and

$$
\Pi_{\kappa}=\partial^{2 \kappa} / \partial x^{2 \kappa}
$$

By owing to the idea of the LFVIM (see [20, 22]), the local fractional functional is determined by

$$
\Theta_{n+1}(x, \tau)=\Theta_{n}(x, \tau)+{ }_{0} I_{\tau}^{(\kappa)}\left\{\frac{\lambda(\eta-\tau)}{\Gamma(1+\kappa)}\left[\mathrm{E}_{\kappa} \Theta_{n}(x, \tau)-\Pi_{\kappa} \Theta_{n}(x, \tau)\right]\right\} .
$$

By taking LFST of 2.2 , we obtain

$$
S_{\kappa}\left\{\Theta_{n+1}(x, \tau)\right\}=S_{\kappa}\left\{\Theta_{n}(x, \tau)\right\}+S_{\kappa}\left\{\frac{\lambda(\eta-\tau)}{\Gamma(1+\kappa)}\right\} S_{\kappa}\left\{\left[\mathrm{E}_{\kappa} \Theta_{n}(x, \tau)-\Pi_{\kappa} \Theta_{n}(x, \tau)\right]\right\},
$$

where

$$
S_{\kappa}\left\{\frac{d^{\kappa} \Theta(\tau)}{d \tau^{\kappa}}\right\}=\frac{1}{h^{\kappa}}\left[S_{\kappa}\{\Theta(\tau)\}-\Theta(0)\right] .
$$

By adopting local fractional variation (see [1, 3, 22]), we can rewrite 2.3 as

$$
\delta^{\kappa}\left\{\Theta_{n+1}(x, \tau)\right\}=\delta^{\kappa}\left\{S_{\kappa}\left\{\Theta_{n}(x, \tau)\right\}\right\}+\delta^{\kappa}\left\{S_{\kappa}\left\{\frac{\lambda(\eta-\tau)}{\Gamma(1+k)}\right\} S_{\kappa}\left[\mathrm{E}_{\kappa} \Theta_{n}(x, \tau)-\Pi_{\kappa} \Theta_{n}(x, \tau)\right]\right\} .
$$

Thus we find from 2.5 that

$$
\delta^{\kappa}\left\{S_{\kappa}\left\{\Theta_{n+1}(x, \tau)\right\}\right\}=\delta^{\kappa}\left\{S_{\kappa}\left\{\Theta_{n}(x, \tau)\right\}\right\}+\delta^{\kappa}\left\{S_{\kappa}\left\{\frac{\lambda(\eta-\tau)}{\Gamma(1+k)}\right\} S_{\kappa}\left[\mathrm{E}_{\kappa} \Theta_{n}(x, \tau)\right]\right\}=0 .
$$

In view of 2.6, we have

$$
\delta^{\kappa}\left\{S_{\kappa}\left[\mathrm{E}_{\kappa} \Theta_{n}(x, \tau)\right]\right\}=\delta^{\kappa}\left\{\frac{1}{h^{\kappa}} S_{\kappa}\left\{\Theta_{n}(x, \tau)-\Theta_{n}(x, 0)\right\}\right\}=\frac{1}{h^{\kappa}} \delta^{\kappa} S_{\kappa}\left\{\Theta_{n}(x, \tau)\right\},
$$

such that

$$
1+S_{\kappa}\left\{\frac{\lambda(\tau)}{\Gamma(1+\kappa)}\right\} \frac{1}{h^{\kappa}}=0 .
$$

By using 2.7), we have

$$
S_{\kappa}\left\{\frac{\lambda(\tau)}{\Gamma(1+\kappa)}\right\}=-h^{\kappa},
$$

such that the local fractional iteration algorithm is expressed by

$$
S_{\kappa}\left\{\Theta_{n+1}(x, \tau)\right\}=S_{\kappa}\left\{\Theta_{n}(x, \tau)\right\}-h^{\kappa} S_{\kappa}\left\{\left[\mathrm{E}_{\kappa} \Theta_{n}(x, \tau)-\Pi_{\kappa} \Theta_{n}(x, \tau)\right]\right\} .
$$

Therefore, the solution of (2.1) takes the following form

$$
S_{\kappa}\{\Theta(x, \tau)\}=\lim _{n \rightarrow \infty} S_{\kappa}\left\{\Theta_{n}(x, \tau)\right\} .
$$

By taking the inverse LFST, we find from 2.9 that

$$
\Theta(x, \tau)=S_{\kappa}^{-1}\left\{\lim _{n \rightarrow \infty} S_{\kappa}\left\{\Theta_{n}(x, \tau)\right\}\right\} .
$$

\section{Solving local fractional diffusion equation in fractal one-dimensional space}

In this section, an illustrative example is presented.

We begin with the following local fractional diffusion equation in fractal one-dimensional space (see [3, [5, 18])

$$
\frac{\partial^{\kappa} \Theta(x, t)}{\partial \tau^{\kappa}}-\frac{\partial^{2 \kappa} \Theta(x, t)}{\partial x^{2 \kappa}}=0, \quad \tau \geq 0, \quad 0<x<\pi,
$$

subject to the initial-boundary value conditions given by 


$$
\begin{aligned}
& \Theta(x, 0)=\sin _{\kappa}\left(x^{\kappa}\right) \quad 0<x<\pi \\
& \Theta(\pi, \tau)=0, \quad \tau \geq 0 \\
& \Theta(0, \tau)=0, \quad \tau \geq 0
\end{aligned}
$$

where

$$
\sin _{\kappa}\left(x^{\kappa}\right)=\sum_{i=0}^{\infty}(-1)^{\kappa} \frac{x^{(2 i+1) \kappa}}{\Gamma(1+(2 i+1) \kappa)},
$$

represents the special function defined on Cantor sets (see [12, 22]) and the corresponding graph is demonstrated in Figure 1.

In view of $(2.8)$, we have

$$
S_{\kappa}\left\{\Theta_{n+1}(x, \tau)\right\}=S_{\kappa}\left\{\Theta_{n}(x, \tau)\right\}-h^{\kappa} S_{\kappa}\left\{\left[\mathrm{E}_{\kappa} \Theta_{n}(x, \tau)-\Pi_{\kappa} \Theta_{n}(x, \tau)\right]\right\},
$$

and, alternatively,

$$
\Theta_{n+1}(h, \tau)=\Theta_{n}(h, \tau)-h^{\kappa} S_{\kappa}\left\{\left[\mathrm{E}_{\kappa} \Theta_{n}(x, \tau)-\Pi_{\kappa} \Theta_{n}(x, \tau)\right]\right\},
$$

with the initial-value condition given by

$$
\Theta_{0}(x, h)=S_{\kappa}\left\{\Theta_{0}(x, 0)\right\}=\sin _{\kappa}\left(x^{\kappa}\right) .
$$

Thus, we have the following result from 3.2 and 3.3

$$
\begin{aligned}
\Theta_{1}(h, \tau) & =\Theta_{0}(h, \tau)-h^{\kappa} S_{\kappa}\left\{\left[\mathrm{E}_{\kappa} \Theta_{0}(x, \tau)-\Pi_{\kappa} \Theta_{0}(x, \tau)\right]\right\} \\
& =\Theta_{0}(h, \tau)-h^{\kappa}\left\{\frac{1}{h^{\kappa}}\left[\Theta_{0}(x, h)-\Theta_{0}(x, 0)\right]-\Pi_{\kappa} \Theta_{0}(x, h)\right\} \\
& =\sin _{\kappa}\left(x^{\kappa}\right) \sum_{i=0}^{1}(-1)^{i} h^{i \kappa}, \\
\Theta_{2}(h, \tau) & =\Theta_{1}(h, \tau)-h^{\kappa} S_{\kappa}\left\{\left[\mathrm{E}_{\kappa} \Theta_{1}(x, \tau)-\Pi_{\kappa} \Theta_{1}(x, \tau)\right]\right\} \\
& =\Theta_{1}(h, \tau)-h^{\kappa}\left\{\frac{1}{h^{\kappa}}\left[\Theta_{1}(x, h)-\Theta_{1}(x, 0)\right]-\Pi_{\kappa} \Theta_{1}(x, h)\right\} \\
& =\sin _{\kappa}\left(x^{\kappa}\right) \sum_{i=0}^{2}(-1)^{i} h^{i \kappa}, \\
\Theta_{3}(h, \tau) & =\Theta_{2}(h, \tau)-h^{\kappa} S_{\kappa}\left\{\left[\mathrm{E}_{\kappa} \Theta_{2}(x, \tau)-\Pi_{\kappa} \Theta_{2}(x, \tau)\right]\right\} \\
& =\Theta_{2}(h, \tau)-h^{\kappa}\left\{\frac{1}{h^{\kappa}}\left[\Theta_{2}(x, h)-\Theta_{2}(x, 0)\right]-\Pi_{\kappa} \Theta_{2}(x, h)\right\} \\
& =\sin _{\kappa}\left(x^{\kappa}\right) \sum_{i=0}^{3}(-1)^{i} h^{i \kappa},
\end{aligned}
$$

and so on.

Consequently, we have

$$
\Theta_{n}(h, \tau)=\lim _{n \rightarrow \infty} \sin _{\kappa}\left(x^{\kappa}\right) \sum_{i=0}^{n}(-1)^{i} h^{i \kappa} .
$$

Therefore, by taking the inverse LFST of (3.4), we obtain

$$
\Theta(x, \tau)=S_{\kappa}^{-1}\left\{\lim _{n \rightarrow \infty} S_{\kappa}\left\{\Theta_{n}(x, \tau)\right\}\right\}
$$




$$
\begin{aligned}
& =S_{\kappa}^{-1}\left\{\lim _{n \rightarrow \infty} S_{\kappa}\left\{\sin _{\kappa}\left(x^{\kappa}\right) \sum_{i=0}^{n}(-1)^{i} h^{i \kappa}\right\}\right\} \\
& =S_{\kappa}^{-1}\left\{\lim _{n \rightarrow \infty} S_{\kappa}\left\{\sin _{\kappa}\left(x^{\kappa}\right) \frac{1}{1+h^{\kappa}}\right\}\right\} \\
& =\sin _{\kappa}\left(x^{\kappa}\right) E_{\kappa}\left(-\tau^{\kappa}\right),
\end{aligned}
$$

and the exact solution of (3.1) is shown in Figure 2, where

$$
S_{\kappa}\left\{E_{\kappa}\left(-\tau^{\kappa}\right)\right\}=\frac{1}{1-h^{\kappa}},
$$

with the following Mittag-Leffler function on Cantor sets [22]:

$$
E_{\kappa}\left(\tau^{\kappa}\right)=\sum_{i=0}^{\infty} \frac{\tau^{i \kappa}}{\Gamma(1+i \kappa)},
$$

and the corresponding graph when $\kappa=\ln 2 / \ln 3$ represented in Figure 3 .

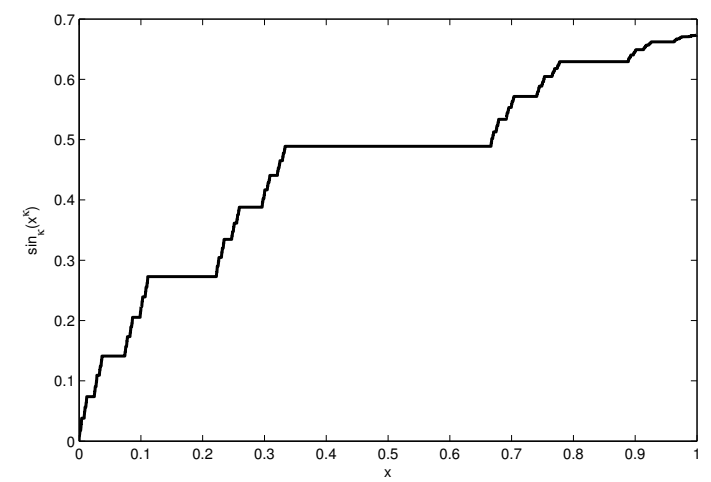

Figure 1: The special function defined on Cantor sets when $\kappa=\ln 2 / \ln 3$.

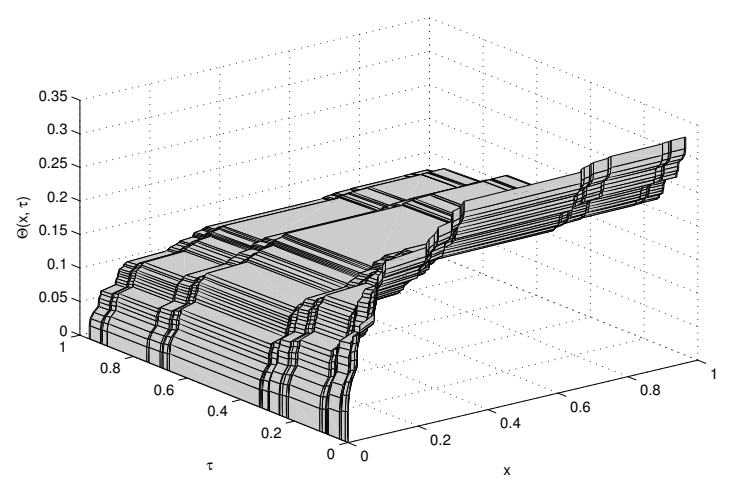

Figure 2: The non-differentiable solution for the local fractional diffusion equation when $\kappa=\ln 2 / \ln 3$.

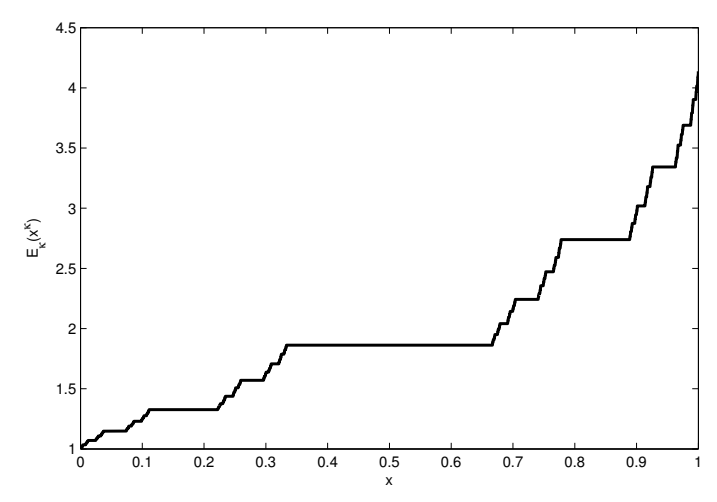

Figure 3: The Mittag-Leffler function on Cantor sets when $\kappa=\ln 2 / \ln 3$.

\section{Conclusions}

The work has presented a coupling method involving the LFVIM with the LFST. The local fractional diffusion equation in fractal one-dimensional space was discussed. The non-differentiable exact solution 
for description of the fractal heat transfer was also obtained. The present methodology is proposed as an accurate and efficient tool for solving the local fractional PDEs in mathematical physics.

\section{Acknowledgment}

This work is supported by the State Key Research Development Program of the People's Republic of China (Grant No. 2016YFC0600705), the Priority Academic Program Development of Jiangsu Higher Education Institutions (PAPD2014) and National Natural Science Foundation of China (Grant No. 51404250).

\section{References}

[1] S. Blasiak, Time-fractional heat transfer equations in modeling of the non-contacting face seals, Int. J. Heat Mass Transf., 100 (2016), 79-88. 1, 2

[2] L. Chen, B. Basu, D. McCabe, Fractional order models for system identification of thermal dynamics of buildings, Energy and Buildings, 133 (2016), 381-388.

[3] R. S. Damor, S. Kumar, A. K. Shukla, Solution of fractional bioheat equation in terms of Fox's H-function, SpringerPlus, 5 (2016), 10 pages. 2, 3

[4] M. A. Ezzat, State space approach to thermoelectric fluid with fractional order heat transfer, Heat Mass Transf., 48 (2012), 71-82.

[5] M. A. Ezzat, A. A. El-Bary, Effects of variable thermal conductivity and fractional order of heat transfer on a perfect conducting infinitely long hollow cylinder, Int. J. Thermal Sci., 108 (2016), 62-69. 1, 3

[6] J. Hristov, Heat-balance integral to fractional (half-time) heat diffusion sub-model, Thermal Sci., 14 (2010), 291316. 1

[7] F. Huang, F. Liu, The space-time fractional diffusion equation with Caputo derivatives, J. Appl. Math. Comput., 19 (2005), 179-190. 1

[8] I. S. Jesus, J. A. T. Machado, Fractional control of heat diffusion systems, Nonlinear Dyn., 54 (2008), $263-282$. 1

[9] V. V. Kulish, J. L. Large, Fractional-diffusion solutions for transient local temperature and heat flux, ASME J. Heat Transf., 122 (2000), 372-376. 1

[10] C.-F. Liu, S.-S. Kong, S.-J. Yuan, Reconstructive schemes for variational iteration method within Yang-Laplace transform with application to fractal heat conduction problem, Thermal Sci., 17 (2013), 715-721. 1, 1

[11] L. Liu, L. Zheng, F. Liu, X. Zhang, An improved heat conduction model with Riesz fractional Cattaneo-Christov flux, Int. J. Heat Mass Transf., 103 (2016), 1191-1197. 1

[12] M. Ma, D. Baleanu,Y. S. Gasimov, X.-J. Yang, New results for multidimensional diffusion equations in fractal dimensional space, Rom. J. Phys., 61 (2016), 784-794. 1 , 3

[13] Y. Z. Povstenko, Fractional heat conduction in infinite one-dimensional composite medium, J. Thermal Stresses, 36 (2013), 351-363. 1

[14] G. S. Priya, P. Prakash, J. J. Nieto, Z. Kayar, Higher-order numerical scheme for the fractional heat equation with Dirichlet and Neumann boundary conditions, Numer. Heat Trans., Part B: Fund.: Int. J. Comput. Methodol., 63 (2013), 540-559.

[15] R. Simpson, A. Jaques, H. Nuñez, C. Ramirez, A. Almonacid, Fractional calculus as a mathematical tool to improve the modeling of mass transfer phenomena in food processing, Food Eng. Rev., 5 (2013), 45-55. 1

[16] J. Singh, D. Kumar, J. J. Nieto, A reliable algorithm for a local fractional Tricomi equation arising in fractal transonic flow, Entropy, 18 (2016), 8 pages. 1

[17] H. M. Srivastava, A. K. Golmankhaneh, D. Baleanu, X.-J. Yang, Local fractional Sumudu transform with application to IVPs on Cantor sets, Abstr. Appl. Anal., 2014 (2014), 7 pages. 1

[18] A. Suzuki, Y. Niibori, S. A. Fomin, V. A. Chugunov, T. Hashida, Analysis of water injection in fractured reservoirs using a fractional-derivative-based mass and heat transfer model, Math. Geosci., 47 (2015), 31-49. 13

[19] X.-J. Yang, Advanced local fractional calculus and its applications, World Science Publisher, New York, (2012). 1

[20] X.-J. Yang, D. Baleanu, Fractal heat conduction problem solved by local fractional variation iteration method, Thermal Sci., 17 (2013), 625-628. 1, 1, 2

[21] X.-J. Yang, D. Baleanu, H. M. Srivastava, Local fractional similarity solution for the diffusion equation defined on Cantor sets, Appl. Math. Lett., 47 (2015), 54-60. 1

[22] X.-J. Yang, D. Baleanu, H. M. Srivastava, Local fractional integral transforms and their applications, Elsevier/Academic Press, Amsterdam, (2016). 1, 1, 2, 2, 3, 3

[23] X.-J. Yang, H. M. Srivastava, An asymptotic perturbation solution for a linear oscillator of free damped vibrations in fractal medium described by local fractional derivatives, Commun. Nonlinear Sci. Numer. Simul., 29 (2015), 499-504. 1 